\title{
Immersion and Invariance Adaptive Backstepping Spacecraft Attitude Control with Modified Rodrigues Parameters
}

\author{
Guilherme F. Trigo and Qi-Ping Chu
}

\begin{abstract}
An adaptive Backstepping attitude control law based on Immersion and Invariance (I\&I) is developed for the TU Delft FAST-D micro-satellite. Tackling both system uncertainties and disturbances, a general additively disturbed rotational dynamics model is considered. In the I\&I framework the parameters to be estimated are most commonly constant. Here, however, the family of I\&I-Backstepping controllers is shown to be input-to-state stable for a class of strict-feedback systems with time-varying uncertainties. This novel result allows the design of an I\&I attitude controller for the spacecraft model considered. The developed control law shows superior performance with respect to Standard (non-adaptive) and Tuning Functions adaptive Backstepping controllers under nominal and heavily perturbed conditions.
\end{abstract}

\section{Introduction}

The ever-wider span of satellite applications brought a generalized demand for smaller and cheaper spacecraft. Smaller scale, however, carries higher vulnerability to disturbances and lower power capacity, reinforcing the need for increasingly robust and efficient Attitude Control Systems (ACSs). In 2007, Delft University of Technology and Beijing's Tsinghua University joined efforts and started the Formation for Atmospheric Science and Technology demonstration (FAST) [17, 5, 6]. The mission consists of the formation flying of two micro-satellites that will use cutting edge technology to collect high atmospheric data. Due to its academic nature, and

G. F. Trigo

German Aerospace Center (DLR), Institute of Space Systems, GNC Systems Department, Bremen, Germany, e-mail: guilherme.trigo@dlr.de

Q. P. Chu

Delft University of Technology, Faculty of Aerospace Engineering, Control and Simulation Department, Delft, The Netherlands 
adding to the limitations and vulnerabilities of small size/weight, the ACS of FAST$\mathrm{D}$, the Dutch element of the swarm, faces several challenges such as high pointing accuracy and stability requirements, limited precision of inertial information, and possible moving parts among carried instruments.

The most used solution in practice for such attitude control problem involves the application of linear robust laws in a scheduled manner, resulting in a limited operation envelope in which the system has conservative and uneven performance [13]. Alternatively, nonlinear control laws provide (in most cases) full-envelope control with consistent performance. However, for relying deeply on the correct modeling of the system, the most used nonlinear laws are particularly vulnerable to disturbances or unmodeled dynamics. Adaptive schemes are a common remedy in this case. Due to their strong stability basis, Lyapunov-based control laws (as the Backstepping [14]) lend themselves particularly well to adaptive feedback.

Adaptive Backstepping by Tuning Functions is a well-known technique in which control and adaptation are derived together following Lyapunov stability conditions [9]. Despite their stability properties, these laws are hard to tune as faster adaptation does not necessarily yield better transient performance. Li et al. [16] applied this method to control the attitude of a spacecraft with uncertain inertia.

A different approach combines, in an adaptive modular scheme, the Backtepping control law with the estimate given by a non-Lyapunov identifier such as the recursive least-squares identifier [25] or the high-gain observer [2]. Since the certainty equivalence principle does usually hold for nonlinear systems, this configuration requires the addition of nonlinear damping terms for robustness against estimation error growth [15]. This emend, however, can yield undesirably high gain control.

A recently developed Lyapunov-based adative control method employs the Immersion and Invariance (I\&I) theory $[1,10,11,12]$. In this framework the estimation error can be assigned a stable dynamics, making it easier to tune than the Tuning Function in the traditional adaptive Backstepping. The estimation law obtained with this method can be with a Backstepping controller as it was done by Sonneveldt et al. [22] to adaptively control a fighter aircraft model.

In this contribution, we show that the I\&I adaptive Backstepping approach is input-to-state stable for a class of strict-feedback systems with time-varying parametric uncertainties. This stability result allows for a rather general description of the spacecraft uncertainties in the design, as a sum of potentially time-variant disturbance torques. The proposed I\&I adaptive attitude controller for this model is derived using command filters which not only simplifies the concept but also provides means of limiting the control signals [28, 3]. Use of a three-element parametrization as the Modified Rodrigues Parameters further simplifies the design in comparison to a quaternion-based one.

We start by introducing the spacecraft model used for the control design. Then, the concept of Immersion and Invariance and its application to adaptive Backstepping are briefly explained. The stability proof of such a control law for a class of systems with varying uncertainties is then shown. Coming to the attitude control design, first a baseline Standard Backstepping attitude controller is constructed, which then is used to draw the I\&I adaptive Backstepping attitude control law. A Tuning 
Functions adaptive Backstepping law is also derived. Finally, comparative testing of the three controllers is carried out for nominal and disturbed scenarios.

\section{Spacecraft Model}

\subsection{Modified Rodrigues Parameters}

The Modified Rodrigues Parameters (MRP) are a three-element attitude representation obtained by stereographical projection of the sphere of quaternion parameters $[21,20]$. In its positive form, with $(0,0,0,-1)$ as point of infinity, the MRP vector $\boldsymbol{\sigma}$ is given by

$$
\boldsymbol{\sigma}=\frac{\mathbf{q}_{1: 3}}{1+\mathrm{q}_{4}},
$$

where $\mathbf{q}_{1: 3}$ is the quaternion vector part and $\mathrm{q}_{4}$ is the scalar part. This transformation makes the MRP representation the most near-linearly behaving three-component attitude parameterization [8]. The Direction Cosine Matrix (DCM) corresponding to the rotation represented by $\boldsymbol{\sigma}$ is given by

$$
\mathbf{C}(\boldsymbol{\sigma})=\mathbf{I}-4 \frac{1-|\boldsymbol{\sigma}|^{2}}{\left(1+|\boldsymbol{\sigma}|^{2}\right)^{2}}[\boldsymbol{\sigma} \times]+\frac{8}{\left(1+|\boldsymbol{\sigma}|^{2}\right)^{2}}[\boldsymbol{\sigma} \times]^{2},
$$

where $[\boldsymbol{\sigma} \times]$ is a skew-symmetric matrix of $\boldsymbol{\sigma}$ and $\mathbf{I}$ is a $3 \times 3$ identity matrix. The MRP kinematics is written

$$
\dot{\boldsymbol{\sigma}}=\mathbf{B}(\boldsymbol{\sigma}) \boldsymbol{\omega},
$$

with

$$
\mathbf{B}(\boldsymbol{\sigma})=\frac{1}{2}[\boldsymbol{\sigma} \times]+\frac{1}{4}\left(1-|\boldsymbol{\sigma}|^{2}\right) \mathbf{I}+\frac{1}{2} \boldsymbol{\sigma} \boldsymbol{\sigma}^{\top},
$$

where $\boldsymbol{\omega}$ is the angular velocity between the reference frames selected for the definition of the attitude.

The following identities will be useful when defining the control laws later on

$$
\begin{gathered}
\mathbf{B}(\boldsymbol{\sigma}) \mathbf{C}(\boldsymbol{\sigma})=\mathbf{B}(-\boldsymbol{\sigma}) \\
\mathbf{C}(\boldsymbol{\sigma}) \boldsymbol{\sigma}=\boldsymbol{\sigma} .
\end{gathered}
$$

\section{Local Vertical Frame and Attitude Kinematics}

Given the Earth observation mission of the spacecraft under study, it is convenient to consider its attitude with respect to a orbital nadir-pointing frame. The LocalVertical Local-Horizontal (LVLH) or Orbital frame, denoted $O$, is centered on the spacecraft CoM, has the $\mathrm{z}$-axis pointing to the center of the Earth, the $\mathrm{y}$-axis pointing 
in the negative direction of the orbital angular momentum, and the $\mathrm{x}$-axis completing the right-handed frame.

The attitude of the spacecraft Body-fixed reference frame $B$ with respect to this Orbital frame is given by $\boldsymbol{\sigma}_{O}^{B}$, follows a kinematics law of the form of (3)

$$
\dot{\boldsymbol{\sigma}}_{O}^{B}=\mathbf{B}\left(\boldsymbol{\sigma}_{O}^{B}\right) \boldsymbol{\omega}_{B O}^{B},
$$

where the body angular velocity with respect to the Orbital frame can be split as

$$
\boldsymbol{\omega}_{B O}^{B}=\boldsymbol{\omega}_{B I}^{B}-\mathbf{C}_{O}^{B} \mathbf{n}_{O I}^{O} .
$$

$\boldsymbol{\omega}_{B I}^{B}$ is the absolute angular velocity of the spacecraft written in Body coordinates and $\mathbf{n}_{O I}^{O}$ the angular velocity vector of the Orbital frame. The latter is given in terms of the orbital rate value, $n_{\mathrm{o}}$, as $\mathbf{n}_{O I}^{O}=\left[\begin{array}{lll}0 & -n_{\mathrm{o}} & 0\end{array}\right]^{\top}$. The direction cosine matrix $\mathbf{C}_{O}^{B}$ from Orbital to Body frame is computed from $\boldsymbol{\sigma}_{O}^{B}$ using Expression (2) as

$$
\mathbf{C}_{O}^{B} \triangleq \mathbf{C}\left(\boldsymbol{\sigma}_{O}^{B}\right) .
$$

\section{Attitude Error Kinematics}

The attitude error parameter $\boldsymbol{\delta} \boldsymbol{\sigma}$ is here defined as representing the rotation from the desired attitude $\boldsymbol{\sigma}_{O, \mathrm{r}}^{B}$ to the actual one $\boldsymbol{\sigma}_{O}^{B}$, i.e.

$$
\boldsymbol{\sigma}_{O}^{B}=\boldsymbol{\delta} \boldsymbol{\sigma} \otimes \boldsymbol{\sigma}_{O, \mathrm{r}}^{B}
$$

where $\otimes$ denotes MRP composition ${ }^{1}$.

Inverting (10) yields

$$
\boldsymbol{\delta} \boldsymbol{\sigma}=\boldsymbol{\sigma}_{O}^{B} \otimes\left(-\boldsymbol{\sigma}_{O, \mathrm{r}}^{B}\right)=\frac{\left(1-\left|\boldsymbol{\sigma}_{\mathrm{r}}\right|^{2}\right) \boldsymbol{\sigma}-\left(1-|\boldsymbol{\sigma}|^{2}\right) \boldsymbol{\sigma}_{\mathrm{r}}+2 \boldsymbol{\sigma} \times \boldsymbol{\sigma}_{\mathrm{r}}}{1+|\boldsymbol{\sigma}|^{2}\left|\boldsymbol{\sigma}_{\mathrm{r}}\right|^{2}+2 \boldsymbol{\sigma}^{\top} \boldsymbol{\sigma}_{\mathrm{r}}} .
$$

where the reference frame super/subscripts were omitted for simplicity.

The MRP error kinematics follows

$$
\boldsymbol{\delta} \dot{\boldsymbol{\sigma}}=\mathbf{B}(\boldsymbol{\delta} \boldsymbol{\sigma}) \boldsymbol{\delta} \boldsymbol{\omega},
$$

with angular velocity error given as

$$
\boldsymbol{\delta} \boldsymbol{\omega}=\boldsymbol{\omega}_{B I}^{B}-\mathbf{C}\left(\boldsymbol{\sigma}_{O}^{B}\right) \mathbf{n}_{O I}^{O}-\mathbf{C}(\boldsymbol{\delta} \boldsymbol{\sigma}) \boldsymbol{\omega}_{B O, \mathrm{r}}^{B},
$$

where $\boldsymbol{\omega}_{B O, \mathrm{r}}^{B}$ is the reference angular velocity.

As it will become evident later on, the error kinematics given in the standard kinematics form by (12) will ease the controller derivation when compared to a partial derivative differential equation form.

\footnotetext{
${ }^{1}$ The rotation represented by $\boldsymbol{\sigma}_{\mathrm{a}} \otimes \boldsymbol{\sigma}_{\mathrm{b}}$ is equivalent to that given by $\mathbf{C}\left(\boldsymbol{\sigma}_{\mathrm{a}}\right) \mathbf{C}\left(\boldsymbol{\sigma}_{\mathrm{b}}\right)$.
} 


\subsection{Attitude Dynamics}

Considering the spacecraft a rigid-body, the body attitude dynamics with respect to an inertial frame $I$ is given by [26]

$$
\mathbf{J}^{B} \dot{\boldsymbol{\omega}}_{B I}^{B}=-\mathbf{\Omega}_{B I}^{B} \mathbf{h}_{B I}^{B}+\mathbf{m}^{B}
$$

where $\boldsymbol{\Omega}_{B I}^{B}$ is the skew symmetric matrix of $\boldsymbol{\omega}_{B I}^{B}, \mathbf{h}_{B I}^{B}=\mathbf{J}^{B} \boldsymbol{\omega}_{B I}^{B}$ is the total angular momentum, $\mathbf{J}^{B}$ is the inertia tensor and $\mathbf{m}^{B}$ is the sum of all external torques applied to the spacecraft. This latter term is considered to include a gravity gradient induced torque $\mathbf{m}_{\mathrm{g}}^{B}$, a control input torque $\mathbf{m}_{\mathrm{c}}^{B}$ and an unknown (potentially time-variant) disturbance torque $\mathbf{m}_{\mathrm{d}}^{B}$,

$$
\mathbf{m}^{B}=\mathbf{m}_{\mathrm{g}}^{B}+\mathbf{m}_{\mathrm{c}}^{B}+\mathbf{m}_{\mathrm{d}}^{B} .
$$

The gravity gradient induced torque is modeled as [27]

$$
\mathbf{m}_{\mathrm{g}}^{B}=3 n_{\mathrm{o}}^{2}\left[\mathbf{c}_{3}\left(\boldsymbol{\sigma}_{O}^{B}\right) \times\right] \mathbf{J}^{B} \mathbf{c}_{3}\left(\boldsymbol{\sigma}_{O}^{B}\right)
$$

where $\mathbf{c}_{3}\left(\boldsymbol{\sigma}_{O}^{B}\right)$ is the third column of the direction cosine matrix (9).

\section{Immersion and Invariance Adaptive Control}

This section presents the Immersion and Invariance (I\&I) framework, first proposed by [1], and its combination with Backstepping control methodology. After a brief introduction to the concept of I\&I, the general design logic of an I\&I-based adaptive Backstepping controller is explained. For a more detailed version of the text presented in this section please refer to [23].

The name of the I\&I framework comes from the fact that the design method uses an immersion of the system into the desired dynamics together with the definition of an invariant manifold. It can be used to adaptively stabilize systems with uncertain parameters. To illustrate how, consider the scalar system

$$
\dot{x}=f(x)+g(x) u,
$$

where $x \in \mathbb{R}, u \in \mathbb{R}$ and the vector function $f(x)$ depends linearly on the unknown parameter $\theta \in \mathbb{R}$ as

$$
f(x)=f_{0}(x)+f_{\theta}(x) \theta,
$$

for some known functions $f_{0}(x): \mathbb{R} \rightarrow \mathbb{R}$ and $f_{\theta}(x): \mathbb{R} \rightarrow \mathbb{R}$. Assume, in a certainty equivalence manner, that there exists a full-information control law $u=v(x, \theta)$ such that the closed-loop system

$$
\dot{x}=f_{*}(x)=f(x)+g(x) v(x, \theta)
$$

has a globally asymptotically stable equilibrium at $x=x_{*}$. 
The problem is solved by finding $\beta(x)$ and $w(x, \hat{\theta})$ such that all trajectories of the extended system

$$
\left\{\begin{array}{l}
\dot{x}=f(x)+g(x) v(x, \hat{\theta}+\beta(x)) \\
\dot{\hat{\theta}}=w(x, \hat{\theta})
\end{array}\right.
$$

are bounded and satisfy

$$
\lim _{t \rightarrow \infty}[g(x) v(x, \hat{\theta}+\beta(x))-g(x) v(x, \theta)]=0 .
$$

If such task is possible, system (17) is said to be Adaptively I\&I Stabilizable.

Condition (21) is satisfied for all trajectories converging to the manifold

$$
\mathcal{M}=\left\{(x, \hat{\theta}) \in \mathbb{R}^{2} \mid \hat{\theta}-\theta+\beta(x)=0\right\},
$$

i.e. for $\mathcal{M}$ attractive.

Note that, despite departing from a certainty equivalence philosophy, the controller developed by I\&I method does not follow the certainty equivalence principle, in the sense that the parameter estimate is not applied directly to the full-information feedback law. Instead, the true parameter $\theta$ is replaced by $\hat{\theta}+\beta(x)$, where the function $\beta(x)$ adds a "proportional" action to the "integral" action of the estimate update law. For so, this kind of adaptive scheme is also known as nonlinear PI adaptation.

\subsection{I\&I-based Adaptive Backstepping Control}

The Immersion and Invariance control design principles introduced can be synthesized for the adaptive controller design for $n^{\text {th }}$ order parametric strict-feedback systems. Consider a system of the form

$$
\begin{aligned}
\dot{x}_{1} & =f_{1}\left(x_{1}\right)+g_{1}\left(x_{1}\right) x_{2}+\varphi_{1}\left(x_{1}\right) \theta_{1} \\
\dot{x}_{2} & =f_{2}\left(x_{1}, x_{2}\right)+g_{2}\left(x_{1}, x_{2}\right) x_{3}+\varphi_{2}\left(x_{2}\right) \theta_{2} \\
& \vdots \\
\dot{x}_{n} & =f_{n}\left(x_{1}, x_{2}, \ldots, x_{n}\right)+g_{n}\left(x_{1}, x_{2}, \ldots, x_{n}\right) u+\varphi_{n}\left(x_{n}\right) \theta_{n}
\end{aligned}
$$

where $u \in \mathbb{R}$, the functions $g_{i}\left(x_{1}, \ldots, x_{i}\right) \neq 0$ for any $\left(x_{1}, \ldots, x_{n}\right) \in \mathbb{R}^{n}, \varphi_{i}\left(x_{i}\right): \mathbb{R} \rightarrow \mathbb{R}$ are known smooth nonlinear functions and $\theta_{i} \in \mathbb{R}$ are constant unknown parameters. The goal is to make $x_{1}$ asymptotically track a sufficiently smooth reference $y_{\mathrm{r}}$.

\section{Estimator Design}

The first step is the design of an observer for the constant uncertainties $\theta_{i}$. To this end, define the off-the-manifold (error) coordinates 


$$
\eta_{i}=\hat{\theta}_{i}-\theta_{i}+\beta_{i}\left(x_{i}\right), \quad i \in\{1,2, \ldots, n\}
$$

where the estimates $\hat{\theta}_{i}$ are the estimator states and $\beta_{i}\left(x_{i}\right): \mathbb{R} \rightarrow \mathbb{R}$ are smooth functions that will be defined later on. The error dynamics are given by

$$
\begin{aligned}
\dot{\eta}_{i} & =\dot{\hat{\theta}}_{i}+\frac{\partial \beta_{i}}{\partial x_{i}}\left(f_{i}+g_{i} x_{i+1}+\varphi_{i} \theta_{i}\right) \\
& =\dot{\hat{\theta}}_{i}+\frac{\partial \beta_{i}}{\partial x_{i}}\left(f_{i}+g_{i} x_{i+1}+\varphi_{i}\left(\hat{\theta}_{i}+\beta_{i}-\eta_{i}\right)\right)
\end{aligned}
$$

where $x_{n+1} \triangleq u$. The update law $\dot{\hat{\theta}}_{i}$ can be define in such a way that it cancels the known parts of (25). This can be done by writing

which yields

$$
\dot{\hat{\theta}}_{i}=-\frac{\partial \beta_{i}}{\partial x_{i}}\left(f_{i}+g_{i} x_{i+1}+\varphi_{i}\left(\hat{\theta}_{i}+\beta_{i}\right)\right)
$$

$$
\dot{\eta}_{i}=-\frac{\partial \beta_{i}}{\partial x_{i}} \varphi_{i} \eta_{i}
$$

The error system (27) for $i=1,2, \ldots, n$ can be seen as linear time-varying, requiring for stability that the diagonal blocks in the dynamic matrix are rendered negative semi-definite. A choice of $\beta_{i}$ that results in such is

$$
\beta_{i}=\gamma_{i} \int_{0}^{x_{i}} \varphi_{i}(\chi) d \chi, \quad \gamma_{i}>0
$$

Lemma 1. The error system (27), with functions $\beta_{i}$ given as in (28) has a uniformly globally stable equilibrium at the origin. Furthermore, $\eta_{i} \in \mathcal{L}_{\infty}$ and $\varphi_{i} \eta_{i} \in \mathcal{L}_{2}, \forall i \in$ $\{1,2, \ldots, n\}$ and $\forall x_{i}(t)$. Additionally, if the regressor functions $\varphi_{i}$ and their timederivatives are bounded, then $\varphi_{i} \eta_{i} \rightarrow 0$.

Proof: Consider the Lyapunov function $W=\sum_{i=1}^{n} \eta_{i}^{2}$. Its time-derivative along (27) satisfies

$$
\begin{aligned}
\dot{W} & =-2 \sum_{i=1}^{n} \frac{\partial}{\partial x_{i}}\left(\gamma_{i} \int_{0}^{x_{i}} \varphi_{i}(\chi) d \chi\right) \varphi_{i} \eta_{i}^{2} \\
& =-2 \sum_{i=1}^{n} \gamma_{i}\left(\varphi_{i} \eta_{i}\right)^{2}<0, \quad \forall \varphi_{i} \eta_{i} \neq 0
\end{aligned}
$$

Noted that, by Lemma 1 and definition of (24), an estimate is obtained for each $\varphi_{i} \theta_{i}$ of the system (23) instead of $\theta_{i}$. These estimates are given by $\varphi_{i}\left(\hat{\theta}_{i}+\beta_{i}\right)$.

\section{Control Law Design}

Done the general design of the estimator the backstepping control law for I\&I design can now be defined. The design here presented includes command filters. This feature, not only reduces the derivation burden by avoiding the analytic computa- 
tion of the time-derivatives of the virtual input signals, but also allows magnitude and rate constraining of the filtered signals [4].

The controller design starts by defining the tracking error coordinates

$$
z_{i}=x_{i}-x_{i, \mathrm{c}}, \quad i \in\{1,2, \ldots, n\},
$$

with $x_{i, \mathrm{c}} \triangleq y_{\mathrm{r}}$. The signals $x_{i, \mathrm{c}}$ are commanded states that are still to be defined. The inclusion of command filters demands the definition of compensated error coordinates

$$
\bar{z}_{i}=z_{i}-\chi_{i},
$$

where $\chi_{i}$ account for the effect of the command filters and will be defined further in the design. The dynamics of the compensated errors is given by

$$
\dot{\bar{z}}_{i}=\dot{z}_{i}-\dot{\chi}_{i}=f_{i}+g_{i} x_{i+1}+\varphi_{i} \theta_{i}-\dot{x}_{i, \mathrm{c}}-\dot{\chi}_{i},
$$

where $x_{n+1} \triangleq u$. Let the raw control signals be defined as

$$
\begin{aligned}
x_{i+1, \mathrm{c}}^{0} & =g_{i}^{-1}\left(-f_{i}-k_{i} z_{i}-g_{i-1} \bar{z}_{i-1}-\varphi_{i}\left(\hat{\theta}_{i}+\beta_{i}\right)+\dot{x}_{i, \mathrm{c}}\right)-\chi_{i+1}, \quad i \in\{1,2, \ldots, n-1\} \\
u^{0} & =g_{n}^{-1}\left(-f_{n}-k_{n} z_{n}-g_{n-1} \bar{z}_{n-1}-\varphi_{n}\left(\hat{\theta}_{n}+\beta_{n}\right)+\dot{x}_{n, \mathrm{c}}\right)
\end{aligned}
$$

with $k_{i}>0, k_{n}>0, \dot{x}_{1, \mathrm{c}} \triangleq \dot{y}_{\mathrm{r}}, \bar{z}_{0}=0$ and $g_{0}=0$. These signals are low-pass filtered to produce $x_{i+1, \mathrm{c}}$ and $u$ and their derivatives $\dot{x}_{i+1, \mathrm{c}}$ and $\dot{u}$ [3]. The effect of the filter on the error coordinates is estimated by

$$
\begin{aligned}
& \dot{\chi}_{i}=-k_{i} \chi_{i}+g_{i}\left(x_{i+1, \mathrm{c}}-x_{i+1, \mathrm{c}}^{0}\right), \quad i \in\{1,2, \ldots, n-1\} \\
& \dot{\chi}_{n}=-k_{n} \chi_{n}+g_{n}\left(u-u^{0}\right) .
\end{aligned}
$$

Applying (32) and (33) to (31) yields

$$
\begin{aligned}
\dot{\bar{z}}_{i} & =f_{i}+g_{i} z_{i+1}+g_{i}\left(x_{i+1, \mathrm{c}}-x_{i+1, \mathrm{c}}^{0}\right)+g_{i} x_{i+1, \mathrm{c}}^{0}+\varphi_{i} \theta_{i}-\dot{x}_{i, \mathrm{c}}-\dot{\chi}_{i} \\
& =-k_{i} \bar{z}_{i}-\varphi_{i} \eta_{i}+g_{i} \bar{z}_{i+1}-g_{i-1} \bar{z}_{i-1}, \quad i \in\{1,2, \ldots, n-1\} \\
\dot{\bar{z}}_{n} & =f_{n}+g_{n}\left(u-u^{0}\right)+g_{n} u^{0}+\varphi_{n} \theta_{n}-\dot{x}_{n, \mathrm{c}}-\dot{\chi}_{n} \\
& =-k_{n} \bar{z}_{n}-\varphi_{n} \eta_{n}-g_{n-1} \bar{z}_{n-1} .
\end{aligned}
$$

The stability of the closed-loop system is evaluated defining the Lyapunov function $V=W+\sum_{i=1}^{n} \bar{z}_{i}^{2}$. Its time-derivative along the trajectories of $\dot{\bar{z}}_{i}$ is

$$
\begin{aligned}
\dot{V} & =\dot{W}+2 \sum_{i=1}^{n-1} \bar{z}_{i}\left(-k_{i} \bar{z}_{i}-\varphi_{i} \eta_{i}+g_{i} \bar{z}_{i+1}-g_{i-1} \bar{z}_{i-1}\right)+2 \bar{z}_{n}\left(-k_{n} \bar{z}_{n}-\varphi_{n} \eta_{n}-g_{n-1} \bar{z}_{n-1}\right) \\
& =\dot{W}-2 \sum_{i=1}^{n} k_{i} \bar{z}_{i}^{2}-2 \sum_{i=1}^{n} \bar{z}_{i} \varphi_{i} \eta_{i}+2 \sum_{i=1}^{n-1} \bar{z}_{i} g_{i} \bar{z}_{i+1}-2 \sum_{i=1}^{n-1} \bar{z}_{i} g_{i-1} \bar{z}_{i-1}-2 \bar{z}_{n} g_{n-1} \bar{z}_{n-1} \\
& =\dot{W}-2 \sum_{i=1}^{n} k_{i} \bar{z}_{i}^{2}-2 \sum_{i=1}^{n} \bar{z}_{i} \varphi_{i} \eta_{i}
\end{aligned}
$$


to which the result of Lemma 1 can be applied yielding

$$
\begin{aligned}
\dot{V} & =-2 \sum_{i=1}^{n} k_{i} \bar{z}_{i}^{2}-2 \sum_{i=1}^{n} \bar{z}_{i} \varphi_{i} \eta_{i}-2 \sum_{i=1}^{n} \gamma_{i}\left(\varphi_{i} \eta_{i}\right)^{2} \\
& =-2 \sum_{i=1}^{n} k_{i} \bar{z}_{i}^{2}-2 \sum_{i=1}^{n} \frac{1}{\gamma_{i}}\left(\frac{\bar{z}_{i}}{2}+\gamma_{i} \varphi_{i} \eta_{i}\right)^{2}+\frac{1}{2} \sum_{i=1}^{n} \frac{\bar{z}_{i}^{2}}{\gamma_{i}} \\
& \leq-2 \sum_{i=1}^{n}\left(k_{i}-\frac{1}{4 \gamma_{i}}\right) \bar{z}_{i}^{2}
\end{aligned}
$$

(37) becomes

$$
\bar{k}_{i}=k_{i}-\frac{1}{4 \gamma_{i}}>0
$$

$$
\dot{V} \leq-2 \sum_{i=1}^{n} \bar{k}_{i} \bar{z}_{i}^{2}<0, \quad \forall \bar{z}_{i} \neq 0
$$

proving the global stability of the origin of the compensated tracking errors of system (23) adaptively I\&I controlled by (32) together with the estimator designed in the previous section. By Barbalat's Lemma it is possible to further prove $\lim _{t \rightarrow \infty} \bar{z}_{i}=0$ and $\lim _{t \rightarrow \infty} \varphi_{i} \eta_{i}=0$. If the command filters are designed with a high enough bandwidth the compensation signals $\chi_{i}$ will be small, having a negligible effect on the closed-loop system. Hence, the stability properties of $\bar{z}_{i}$ can, in that case, be extended to the real tracking errors $z_{i}$, rendering global tracking of the reference signal. The formal proof of this statement is achieved using Singular Perturbation Theory by Farrell et al. [3].

\subsection{Stability-proof for Time-varying Uncertainties}

The algorithm described thus far has unique stability properties in the case of constant uncertain parameters. In this section, the stability properties of this law will be shown for time-varying uncertainties in systems of the form of (23). The result of this novel proof greatly widens the application span of I\&I-based adaptive control, by extending its properties to systems with time-varying uncertainties.

Consider that, in system (23), $\theta_{i}=\theta_{i}(t), i \in\{1, \ldots, n\}$, are now scalar smooth functions. The dynamics of the off-the-manifold coordinates is then given by

$$
\dot{\eta}_{i}=\dot{\hat{\theta}}_{i}-\dot{\theta}_{i}+\frac{\partial \beta_{i}}{\partial x_{i}} \dot{x}_{i}
$$

which, maintaining the same update law (26), yields

$$
\dot{\eta}_{i}=-\dot{\theta}_{i}-\frac{\partial \beta_{i}}{\partial x_{i}} \varphi_{i} \eta_{i}
$$


If $\beta_{i}$ is chosen as according to (28), by the result of Lemma 1, the time-derivative of the Lyapunov function $W=\sum_{i=1}^{n} \eta_{i}^{2}$ along the trajectories of (41) is

$$
\dot{W}=-2 \sum_{i=1}^{n} \gamma_{i}\left(\varphi_{i} \eta_{i}\right)^{2}-2 \sum_{i=1}^{n} \eta_{i} \dot{\theta}_{i}
$$

Assume the following condition holds

$$
\left|\varphi_{i}\left(x_{i}\right)\right|^{-1}<\rho_{i}, \quad \forall x_{i} \in \mathbb{R}, \quad \forall i \in\{1, \ldots, n\},
$$

where $\rho_{i}$ is a finite positive constant. Maintaining the control law designed in the previous section, the time-derivative of a control Lyapunov function defined as $V=$ $W+\sum_{i=1}^{n} \bar{z}_{i}^{2}$, recalling (36), is now given by

$$
\begin{aligned}
\dot{V} & =-2 \sum_{i=1}^{n} k_{i} \bar{z}_{i}^{2}-2 \sum_{i=1}^{n} \bar{z}_{i} \varphi_{i} \eta_{i}-2 \sum_{i=1}^{n} \gamma_{i}\left(\varphi_{i} \eta_{i}\right)^{2}-2 \sum_{i=1}^{n} \eta_{i} \dot{\theta}_{i} \\
& =-2 \sum_{i=1}^{n}\left(k_{i}-\frac{1}{2 \gamma_{i}}\right) \bar{z}_{i}^{2}-\sum_{i=1}^{n} \frac{1}{\gamma_{i}}\left(\bar{z}_{i}+\gamma_{i} \varphi_{i} \eta_{i}\right)^{2}-\sum_{i=1}^{n} \frac{1}{\gamma_{i}}\left(\frac{\dot{\theta}_{i}}{\varphi_{i}}+\gamma_{i} \varphi_{i} \eta_{i}\right)^{2}+\sum_{i=1}^{n} \frac{\dot{\theta}_{i}^{2}}{\gamma_{i} \varphi_{i}^{2}} \\
& \leq-2 \sum_{i=1}^{n} \bar{k}_{i} \bar{z}_{i}^{2}+\sum_{i=1}^{n} \frac{\rho_{i}^{2}}{\gamma_{i}}\left\|\dot{\theta}_{i}\right\|_{\infty}^{2},
\end{aligned}
$$

where

$$
\bar{k}_{i}=k_{i}-\frac{1}{2 \gamma_{i}}>0 .
$$

Inequality (44) proves the input-to-state stability of the compensated error coordinates, $\bar{z}_{i}$, with respect to the time-derivative of the uncertainty, $\dot{\theta}_{i}$. Hence, the trajectories of the closed-loop system converge to a compact set around the origin of the error coordinates that depends on $\rho_{i}, \gamma_{i}$ and $\left\|\dot{\theta}_{i}\right\|_{\infty}$. Assuming (43) holds, and knowing that all $\theta_{i}$ have bounded first time-derivative, then such set can be made arbitrarily small by increasing the estimation gain $\gamma_{i}$.

\section{Attitude Controller Design}

In this section the control theory described in the preceding one is applied to the spacecraft model earlier derived. Such model can be represented simply as

$$
\begin{gathered}
\dot{\boldsymbol{\sigma}}_{O}^{B}=\boldsymbol{f}_{\sigma}\left(\boldsymbol{\sigma}_{O}^{B}\right)+\mathbf{B}\left(\boldsymbol{\sigma}_{O}^{B}\right) \boldsymbol{\omega}_{B I}^{B} \\
\mathbf{J}^{B} \dot{\boldsymbol{\omega}}_{B I}^{B}=\boldsymbol{f}_{\omega}\left(\boldsymbol{\sigma}_{O}^{B}, \boldsymbol{\omega}_{B I}^{B}\right)+\mathbf{m}_{\mathrm{c}}+\mathbf{m}_{\mathrm{d}}
\end{gathered}
$$

where $\boldsymbol{f}_{\sigma}\left(\boldsymbol{\sigma}_{O}^{B}\right)$ has the orbital angular velocity contribution to the MRP kinematics (7), and $\boldsymbol{f}_{\omega}\left(\boldsymbol{\sigma}_{O}^{B}, \boldsymbol{\omega}_{B I}^{B}\right)$ contains the Coriolis and gravity gradient induced torques in (14). $\mathbf{m}_{\mathrm{c}}$ is the input torque and $\mathbf{m}_{\mathrm{d}}$ is a time-varying unknown disturbance torque. 
Starting by neglecting the action of disturbances, a baseline Backstepping controller using command filters is designed. Then, such controller is used as the fullinformation law in the Immersion and Invariance adaptive design. Finally, to serve as performance simulation comparison, an integrated adaptive Backstepping controller with tuning functions estimation is designed based on the same baseline law. For more details on the command filters used in these laws please refer to [23].

\subsection{Non-adaptive Backstepping Controller}

Assuming an undisturbed model $\left(\mathbf{m}_{\mathrm{d}}=\mathbf{0}\right)$, a static Backstepping controller using command filters can be designed defining the error coordinates

$$
\mathbf{z}_{1}=\boldsymbol{\delta} \boldsymbol{\sigma} \quad \text { and } \quad \mathbf{z}_{2}=\boldsymbol{\omega}_{B I}^{B}-\mathbf{x}_{2, \mathrm{c}},
$$

which, for the use of such command filters, should corrected by

$$
\overline{\mathbf{z}}_{1}=\mathbf{z}_{1} \otimes\left(-\chi_{1}\right) \quad \text { and } \quad \overline{\mathbf{z}}_{2}=\mathbf{z}_{2}-\boldsymbol{\chi}_{2} .
$$

where $\boldsymbol{\chi}_{1}$ and $\boldsymbol{\chi}_{2}$ shall defined be later on.

A control law as

$$
\begin{array}{rlrl}
\mathbf{x}_{2, \mathrm{c}}^{0} & =-\mathbf{K}_{1}\left(\overline{\mathbf{z}}_{1}+\boldsymbol{\chi}_{1}\right)-\mathbf{B}^{-1}\left(\boldsymbol{\sigma}_{O}^{B}\right) \boldsymbol{f}_{\sigma}\left(\boldsymbol{\sigma}_{O}^{B}\right)+\mathbf{C}\left(\boldsymbol{\chi}_{1}\right) \boldsymbol{\omega}_{B O, \mathrm{r}}^{B}-\boldsymbol{\chi}_{2}, & \mathbf{K}_{1}>0 \\
\mathbf{m}_{\mathrm{c}}^{0} & =-\boldsymbol{f}_{\boldsymbol{\omega}}\left(\boldsymbol{\sigma}_{O}^{B}, \boldsymbol{\omega}_{B I}^{B}\right)-\mathbf{K}_{2} \mathbf{z}_{2}+\mathbf{J}^{B} \dot{\mathbf{x}}_{2, \mathrm{c}}-\overline{\mathbf{z}}_{1}, & & \mathbf{K}_{2}>0
\end{array}
$$

globally asymptotically stabilizes the origin of $\left(\overline{\mathbf{z}}_{1}, \overline{\mathbf{z}}_{2}\right)$ of the undisturbed system (46). To prove this, start by considering a command filter effect on the error coordinates given by the stable systems

$$
\begin{aligned}
\dot{\chi}_{1} & =\mathbf{B}\left(\chi_{1}\right)\left(-\mathbf{K}_{1} \chi_{1}+\mathbf{x}_{2, \mathrm{c}}-\mathbf{x}_{2, \mathrm{c}}^{0}\right) \\
\mathbf{J}^{B} \dot{\chi}_{2} & =-\mathbf{K}_{2} \boldsymbol{\chi}_{2}+\mathbf{m}_{\mathrm{c}}-\mathbf{m}_{\mathrm{c}}^{0},
\end{aligned}
$$

where the raw control signals $\mathbf{x}_{2, \mathrm{c}}^{0}$ and $\mathbf{m}_{\mathrm{c}}^{0}$ are filtered to obtain $\mathbf{x}_{2, \mathrm{c}}$ and $\mathbf{m}_{\mathrm{c}}$ and their time-derivatives. As done in [23], such command filtering also imposes limits on value and rate of these signals.

Based on the result described by Tsiotras [24] the following Lemma will be used to prove the stability properties of the problem at hand.

Lemma 2. The time-derivative of a control Lyapunov function defined as $V=$ $2 \log \left(1+|\boldsymbol{\sigma}|^{2}\right)$ taken along the trajectories of the MRP kinematics $\dot{\boldsymbol{\sigma}}=B(\boldsymbol{\sigma}) \boldsymbol{\omega}$ is given by

$$
\dot{V}=\boldsymbol{\sigma}^{\top} \boldsymbol{\omega}
$$

Proof: This result is obtained simply by taking the time-derivative of $V$ and replacing the MRP kinematics expression, noting that $\boldsymbol{\sigma}^{\top}[\boldsymbol{\sigma} \times]=\mathbf{0}$. 
The compensated error dynamics are written

$$
\begin{aligned}
\dot{\mathbf{z}}_{1} & =\mathbf{B}\left(\overline{\mathbf{z}}_{1}\right)\left(\boldsymbol{\delta} \boldsymbol{\omega}-\mathbf{C}\left(\overline{\mathbf{z}}_{1}\right) \mathbf{B}^{-1}\left(\boldsymbol{\chi}_{1}\right) \dot{\boldsymbol{\chi}}_{1}\right) \\
\dot{\mathbf{z}}_{2} & =\left(\mathbf{J}^{B}\right)^{-1} \boldsymbol{f}_{\boldsymbol{\omega}}\left(\boldsymbol{\sigma}_{O}^{B}, \boldsymbol{\omega}_{B I}^{B}\right)+\mathbf{m}_{\mathrm{c}}-\dot{\mathbf{x}}_{2, \mathrm{c}}-\dot{\boldsymbol{\chi}}_{2} .
\end{aligned}
$$

A control Lyapunov function defined as

$$
V=2 \log \left(1+\left|\overline{\mathbf{z}}_{1}\right|^{2}\right)+\frac{1}{2} \overline{\mathbf{z}}_{2}^{\top} \mathbf{J}^{B} \overline{\mathbf{z}}_{2},
$$

using the result of Lemma 2, has a time-derivative along (52) and (53) of

$$
\begin{aligned}
\dot{V}= & \overline{\mathbf{z}}_{1}^{\top}\left(\boldsymbol{\delta} \boldsymbol{\omega}-\mathbf{C}\left(\overline{\mathbf{z}}_{1}\right) \mathbf{B}^{-1}\left(\boldsymbol{\chi}_{1}\right) \dot{\boldsymbol{\chi}}_{1}\right)+\overline{\mathbf{z}}_{2}^{\top}\left(\boldsymbol{f}_{\omega}\left(\boldsymbol{\sigma}_{O}^{B}, \boldsymbol{\omega}_{B I}^{B}\right)+\mathbf{m}_{\mathrm{c}}-\mathbf{J}^{B} \dot{\mathbf{x}}_{2, \mathrm{c}}-\mathbf{J}^{B} \dot{\chi}_{2}\right) \\
= & \overline{\mathbf{z}}_{1}^{\top}\left(\mathbf{z}_{2}+\mathbf{B}^{-1}\left(\boldsymbol{\sigma}_{O}^{B}\right) \boldsymbol{f}_{\sigma}\left(\boldsymbol{\sigma}_{O}^{B}\right)-\mathbf{C}\left(\overline{\mathbf{z}}_{1}\right) \mathbf{C}\left(\boldsymbol{\chi}_{1}\right) \boldsymbol{\omega}_{O I, \mathrm{r}}^{O}+\mathbf{K}_{1} \boldsymbol{\chi}_{1}+\mathbf{x}_{2, \mathrm{c}}^{0}\right) \\
& +\overline{\mathbf{z}}_{2}^{\top}\left(\boldsymbol{f}_{\boldsymbol{\omega}}\left(\boldsymbol{\sigma}_{O}^{B}, \boldsymbol{\omega}_{B I}^{B}\right)-\mathbf{J}^{B} \dot{\mathbf{x}}_{2, \mathrm{c}}+\mathbf{K}_{2} \boldsymbol{\chi}_{2}+\mathbf{m}_{\mathrm{c}}^{0}\right)
\end{aligned}
$$

plugging in the control laws (49)-(50) yields

$$
\begin{aligned}
\dot{V} & =\overline{\mathbf{z}}_{1}^{\top}\left(\mathbf{z}_{2}+\mathbf{K}_{1} \boldsymbol{\chi}_{1}-\mathbf{K}_{1}\left(\overline{\mathbf{z}}_{1}+\boldsymbol{\chi}_{1}\right)-\boldsymbol{\chi}_{2}\right)+\overline{\mathbf{z}}_{2}^{\top}\left(\mathbf{K}_{2} \boldsymbol{\chi}_{2}-\mathbf{K}_{2} \mathbf{z}_{2}-\overline{\mathbf{z}}_{1}\right) \\
& =-\overline{\mathbf{z}}_{1}^{\top} \mathbf{K}_{1} \overline{\mathbf{z}}_{1}-\overline{\mathbf{z}}_{2}^{\top} \mathbf{K}_{2} \overline{\mathbf{z}}_{2}<0, \quad \forall\left(\overline{\mathbf{z}}_{1}, \overline{\mathbf{z}}_{2}\right) \neq \mathbf{0},
\end{aligned}
$$

which proves the global asymptotic stability of the error origin equilibrium.

\subsection{Immersion and Invariance Adaptive Backstepping Controller}

Considering now the presence of a time-variant smooth disturbance torque $\mathbf{m}_{\mathrm{d}}$ in system (46), the static controller derived in the previous section can be made adaptive through the Immersion and Invariance design method, presented in Section 3.1, by using the feedback law

$$
\mathbf{m}_{\mathrm{c}}^{0}=\mathbf{m}_{\mathrm{c}, \mathrm{BS}}^{0}-\hat{\mathbf{m}}_{\mathrm{d}}-\boldsymbol{\beta}\left(\mathbf{h}_{B I}^{B}\right),
$$

where $\mathbf{~ m}_{\mathrm{c}, \mathrm{BS}}^{0}$ is the non-adaptive Backstepping law (50) and $\boldsymbol{\beta}\left(\mathbf{h}_{B I}^{B}\right)$ is a function yet to be defined. Notice that, since the uncertainty is matched in the dynamics loop, the kinematics feedback law is kept the same.

As earlier described, the I\&I procedure starts by defining an off-the-manifold coordinate

$$
\boldsymbol{\eta}=\hat{\mathbf{m}}_{\mathrm{d}}-\mathbf{m}_{\mathrm{d}}+\boldsymbol{\beta}\left(\mathbf{h}_{B I}^{B}\right),
$$

which, in this case, has the dynamics

$$
\begin{aligned}
\dot{\boldsymbol{\eta}} & =\dot{\mathbf{m}}_{\mathrm{d}}-\dot{\mathbf{m}}_{\mathrm{d}}+\frac{\partial \boldsymbol{\beta}\left(\mathbf{h}_{B I}^{B}\right)}{\partial \mathbf{h}_{B I}^{B}}\left(\boldsymbol{f}_{\omega}\left(\boldsymbol{\sigma}_{O}^{B}, \boldsymbol{\omega}_{B I}^{B}\right)+\mathbf{m}_{\mathrm{c}}+\mathbf{m}_{\mathrm{d}}\right) \\
& =\dot{\mathbf{m}}_{\mathrm{d}}-\dot{\mathbf{m}}_{\mathrm{d}}+\frac{\partial \boldsymbol{\beta}\left(\mathbf{h}_{B I}^{B}\right)}{\partial \mathbf{h}_{B I}^{B}}\left(\boldsymbol{f}_{\omega}\left(\boldsymbol{\sigma}_{O}^{B}, \boldsymbol{\omega}_{B I}^{B}\right)+\left(\mathbf{m}_{\mathrm{c}}-\mathbf{m}_{\mathrm{c}}^{0}\right)+\mathbf{m}_{\mathrm{c}}^{0}+\hat{\mathbf{m}}_{\mathrm{d}}+\boldsymbol{\beta}\left(\mathbf{h}_{B I}^{B}\right)-\boldsymbol{\eta}\right)
\end{aligned}
$$




$$
=\dot{\hat{\mathbf{m}}}_{\mathrm{d}}-\dot{\mathbf{m}}_{\mathrm{d}}+\frac{\partial \boldsymbol{\beta}\left(\mathbf{h}_{B I}^{B}\right)}{\partial \mathbf{h}_{B I}^{B}}\left(-\mathbf{K}_{2} \overline{\mathbf{z}}_{2}+\mathbf{J}^{B} \dot{\boldsymbol{\chi}}_{2}+\mathbf{J}^{B} \dot{\mathbf{x}}_{2, \mathrm{c}}-\overline{\mathbf{z}}_{1}-\boldsymbol{\eta}\right) .
$$

Defining the estimate update law as

renders (59)

$$
\dot{\mathbf{m}}_{\mathrm{d}}=\frac{\partial \beta\left(\mathbf{h}_{B I}^{B}\right)}{\partial \mathbf{h}_{B I}^{B}}\left(\mathbf{K}_{2} \overline{\mathbf{z}}_{2}-\mathbf{J}^{B} \dot{\boldsymbol{\chi}}_{2}-\mathbf{J}^{B} \dot{\mathbf{x}}_{2, c}+\overline{\mathbf{z}}_{1}\right) .
$$

$$
\dot{\boldsymbol{\eta}}=-\dot{\mathbf{m}}_{\mathrm{d}}-\frac{\partial \boldsymbol{\beta}\left(\mathbf{h}_{B I}^{B}\right)}{\partial \mathbf{h}_{B I}^{B}} \boldsymbol{\eta} .
$$

Defining a control Lyapunov function as

$$
V=2 \log \left(1+\left|\overline{\mathbf{z}}_{1}\right|^{2}\right)+\frac{1}{2} \overline{\mathbf{z}}_{2}^{\top} \mathbf{J}^{B} \overline{\mathbf{z}}_{2}+\frac{1}{2} \boldsymbol{\eta}^{\top} \boldsymbol{\eta}
$$

and choosing

$$
\boldsymbol{\beta}\left(\mathbf{h}_{B I}^{B}\right)=\Gamma \mathbf{h}_{B I}^{B}, \quad \boldsymbol{\Gamma}=\boldsymbol{\Gamma}^{\top}>0
$$

results in a time-derivative of $V$

$$
\begin{aligned}
\dot{V}= & -\overline{\mathbf{z}}_{1}^{\top} \mathbf{K}_{1} \overline{\mathbf{z}}_{1}-\overline{\mathbf{z}}_{2}^{\top} \mathbf{K}_{2} \overline{\mathbf{z}}_{2}-\overline{\mathbf{z}}_{2}^{\top} \boldsymbol{\eta}-2 \boldsymbol{\eta}^{\top} \dot{\mathbf{m}}_{\mathrm{d}}-\boldsymbol{\eta}^{\top} \boldsymbol{\Gamma} \boldsymbol{\eta} \\
= & -\overline{\mathbf{z}}_{1}^{\top} \mathbf{K}_{1} \overline{\mathbf{z}}_{1}-\overline{\mathbf{z}}_{2}^{\top}\left(\mathbf{K}_{2}-\frac{1}{2} \boldsymbol{\Gamma}^{-1}\right) \overline{\mathbf{z}}_{2}-\frac{1}{2}\left(\overline{\mathbf{z}}_{2}+\boldsymbol{\Gamma} \boldsymbol{\eta}\right)^{\top} \boldsymbol{\Gamma}^{-1}\left(\overline{\mathbf{z}}_{2}+\boldsymbol{\Gamma} \boldsymbol{\eta}\right) \\
& -\frac{1}{2}\left(\boldsymbol{\eta}+\boldsymbol{\Gamma}^{-1} \dot{\mathbf{m}}_{\mathrm{d}}\right)^{\top} \boldsymbol{\Gamma}\left(\boldsymbol{\eta}+\boldsymbol{\Gamma}^{-1} \dot{\mathbf{m}}_{\mathrm{d}}\right)+\frac{1}{2} \dot{\mathbf{m}}_{\mathrm{d}}^{\top} \boldsymbol{\Gamma}^{-1} \dot{\mathbf{m}}_{\mathrm{d}} \\
\leq & -\overline{\mathbf{z}}_{1}^{\top} \mathbf{K}_{1} \overline{\mathbf{z}}_{1}-\overline{\mathbf{z}}_{2}^{\top} \overline{\mathbf{K}}_{2} \overline{\mathbf{z}}_{2}+\frac{1}{2} \dot{\mathbf{m}}_{\mathrm{d}}^{\top} \boldsymbol{\Gamma}^{-1} \dot{\mathbf{m}}_{\mathrm{d}},
\end{aligned}
$$

which, for

$$
\overline{\mathbf{K}}_{2}=\mathbf{K}_{2}-\frac{1}{2} \Gamma^{-1}>0
$$

proves the input-to-state stability of the pair $\left(\overline{\mathbf{z}}_{1}, \overline{\mathbf{z}}_{2}\right)$, and by proper filter design also of $\left(\mathbf{z}_{1}, \mathbf{z}_{2}\right)$, with respect to $\dot{\mathbf{m}}_{\mathrm{d}}$, for the feedback (49) and (50) with estimate update law (60) and $\boldsymbol{\beta}\left(\mathbf{h}_{B I}^{B}\right)$ function (63). Hence, for bounded $\dot{\mathbf{m}}_{\mathrm{d}}$, the compact set around the origin to which the pair of tracking error coordinates converge to can be made arbitrarily small by increasing the entries of the gain matrix $\Gamma$.

\subsection{Adaptive Backstepping Controller with Tuning Functions}

In order to compare the performance of the I\&I adaptive design a "traditional" adaptive Backstepping controller with tuning function estimation is here developed. This type of control law compensates for the presence of the disturbance term using, for this case, the feedback law

$$
\mathbf{m}_{\mathrm{c}}^{0}=\mathbf{m}_{\mathrm{c}, \mathrm{BS}}^{0}-\hat{\mathbf{m}}_{\mathrm{d}} .
$$

where, again, $\mathbf{m}_{\mathrm{c}, \mathrm{BS}}^{0}$ is the control law (50) of the non-adaptive Backstepping. 
In the definition of the control Lyapunov function the estimation error $\boldsymbol{\delta} \mathbf{m}_{\mathrm{d}}=$ $\mathbf{m}_{\mathrm{d}}-\hat{\mathbf{m}}_{\mathrm{d}}$ is accounted for as

$$
V=2 \log \left(1+\left|\overline{\mathbf{z}}_{1}\right|^{2}\right)+\frac{1}{2} \overline{\mathbf{z}}_{2}^{\top} \overline{\mathbf{z}}_{2}+\frac{1}{2} \boldsymbol{\delta} \mathbf{m}_{\mathrm{d}}^{\top} \boldsymbol{\Lambda}^{-1} \boldsymbol{\delta} \mathbf{m}_{\mathrm{d}}, \quad \boldsymbol{\Lambda}=\boldsymbol{\Lambda}^{\top}>0 .
$$

The time-derivative of such Lyapunov function is

$$
\begin{aligned}
\dot{V}= & -\overline{\mathbf{z}}_{1}^{\top} \mathbf{K}_{1} \overline{\mathbf{z}}_{1}-\overline{\mathbf{z}}_{2}^{\top} \mathbf{K}_{2} \overline{\mathbf{z}}_{2}+\overline{\mathbf{z}}_{2}^{\top} \boldsymbol{\delta} \mathbf{m}_{\mathrm{d}}+\boldsymbol{\delta} \mathbf{m}_{\mathrm{d}}^{\top} \boldsymbol{\Lambda}^{-1} \dot{\mathbf{m}}_{\mathrm{d}}-\boldsymbol{\delta} \mathbf{m}_{\mathrm{d}}^{\top} \boldsymbol{\Lambda}^{-1} \dot{\hat{\mathbf{m}}}_{\mathrm{d}} \\
= & -\overline{\mathbf{z}}_{1}^{\top} \mathbf{K}_{1} \overline{\mathbf{z}}_{1}-\overline{\mathbf{z}}_{2}^{\top} \mathbf{K}_{2} \overline{\mathbf{z}}_{2}+\boldsymbol{\delta} \mathbf{m}_{\mathrm{d}}^{T}\left(\overline{\mathbf{z}}_{2}-\boldsymbol{\Lambda}^{-1} \dot{\hat{\mathbf{m}}}_{\mathrm{d}}\right)-\frac{1}{2}\left(\dot{\mathbf{m}}_{\mathrm{d}}-\boldsymbol{\delta} \mathbf{m}_{\mathrm{d}}\right)^{\top} \boldsymbol{\Lambda}^{-1}\left(\dot{\mathbf{m}}_{\mathrm{d}}-\boldsymbol{\delta} \mathbf{m}_{\mathrm{d}}\right) \\
& +\frac{1}{2} \dot{\mathbf{m}}_{\mathrm{d}}^{\top} \boldsymbol{\Lambda}^{-1} \dot{\mathbf{m}}_{\mathrm{d}}+\frac{1}{2} \boldsymbol{\delta} \mathbf{m}_{\mathrm{d}}^{\top} \boldsymbol{\Lambda}^{-1} \boldsymbol{\delta} \mathbf{m}_{\mathrm{d}} \\
\leq & -\overline{\mathbf{z}}_{1}^{\top} \mathbf{K}_{1} \overline{\mathbf{z}}_{1}-\overline{\mathbf{z}}_{2}^{\top} \mathbf{K}_{2} \overline{\mathbf{z}}_{2}+\frac{1}{2} \dot{\mathbf{m}}_{\mathrm{d}}^{\top} \boldsymbol{\Lambda}^{-1} \dot{\mathbf{m}}_{\mathrm{d}}+\frac{1}{2} \boldsymbol{\delta} \mathbf{m}_{\mathrm{d}}^{\top} \boldsymbol{\Lambda}^{-1} \boldsymbol{\delta} \mathbf{m}_{\mathrm{d}}
\end{aligned}
$$

where inequality (68) is made true through the choice of the estimate update law

$$
\dot{\hat{\mathbf{m}}}_{\mathrm{d}}=\operatorname{Proj}\left(\boldsymbol{\Lambda} \overline{\mathbf{z}}_{2}\right) \text {. }
$$

Notice that, differently from the I\&I design case, here the pair of tracking errors is input-to-state stable with respect to both $\mathbf{m}_{\mathrm{d}}$ and $\boldsymbol{\delta} \mathbf{m}_{\mathrm{d}}$, meaning that, in addition to the smoothness condition of the disturbance torque, the estimation error has to remain bounded. This is achieved by using the Projection operator (Proj) on the estimate update law (69), which is here defined as done by Pomet et al. [18]. Also in contrast with the I\&I framework is the fact that the estimate $\hat{\mathbf{m}}_{\mathrm{d}}$ is not guaranteed to converge to actual $\mathbf{m}_{\mathrm{d}}$, rather providing an integrative action to the rate control.

\section{Simulation and Performance Analysis}

In this section the designed controllers are tested and compared in the presence of two different disturbance torques, separately applied, one produced by a saturated uncontrolled reaction wheel and one originated by an oscillating piece of payload. The disturbance dynamics for each of this cases can be found in [23].

For fairness of comparison, the controller gains $\mathbf{K}_{1}$ and $\mathbf{K}_{2}$ were defined equally for the three laws, $\mathbf{K}_{1}=0.4 \mathbf{I}$ and $\mathbf{K}_{2}=5 \mathbf{I}$. The adaptive gains were chosen (equally) as $\boldsymbol{\Gamma}=20 \mathbf{I}$ and $\boldsymbol{\Lambda}=20 \mathbf{I}$. The laws shall be referred to as: S-BS is the (static) nonadaptive Backstepping controller of Section 4.1, TF-BS refers to the Tuning Funstions adaptive Backstepping law (Section 4.3), and I\&I-BS to the Immersion and Invariance controller in Section 4.2. The angular reference signal used includes a MRP-ramp, a steady-pointing period and a sweeping (or scanning) motion stage, as shows Fig. 1. The performance shall be assessed through the evaluation of two error quantities: the one-angle pointing error, $\theta_{e}=4 \arctan (\|\boldsymbol{\delta} \boldsymbol{\sigma}\|)$, and the norm of the angular velocity tracking error, $\omega_{e}=\left\|\boldsymbol{\omega}_{B I}^{B}-\mathbf{x}_{2, c}\right\|$. The project requirements for the attitude controller's performance are $\theta_{e} \leq 30 \mathrm{arcsec}$ and $\omega_{e} \leq 1 \mathrm{arcsec} / \mathrm{s}$. The spacecraft has the inertia tensor $\mathbf{J}^{B}=\operatorname{diag}([3.083,3.083,2.083]) \mathrm{kgm}^{2}$, and flies in an approximately circular orbit of $650 \mathrm{~km}$ altitude and orbital rate $n_{\mathrm{o}}$ of $1.073 \mathrm{~m} \mathrm{rad} / \mathrm{s}$. The control laws run at a frequency of $10 \mathrm{~Hz}$. 


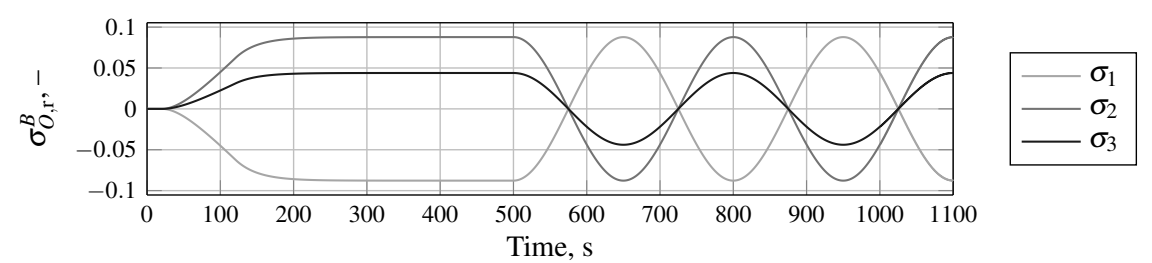

Fig. 1 MRP Attitude reference trajectory.

\section{Nominal Performance}

In a disturbance-free scenario all three controllers succeed in maintaining the tracking errors bellow the required levels (Fig. 2). Table 1 shows the Root-Mean-Square (RMS) pointing error. The non-adaptive and the I\&I laws achieve a similar pointing performance while the tuning functions one lags slightly behind. This is explained by the integrative action of the adaptation law, promoting rate-tracking while compromising pointing accuracy. This is also reflected on the high RMS of the estimated perturbation error in comparison to the I\&I (Table 2). The actuation history is very similar among the three controllers (Fig. 3), being differences only perceived when computing the total workload (Table 3). This reveals a slightly higher effort by the TF-BS law comparing to the other two laws, which yield identical results.
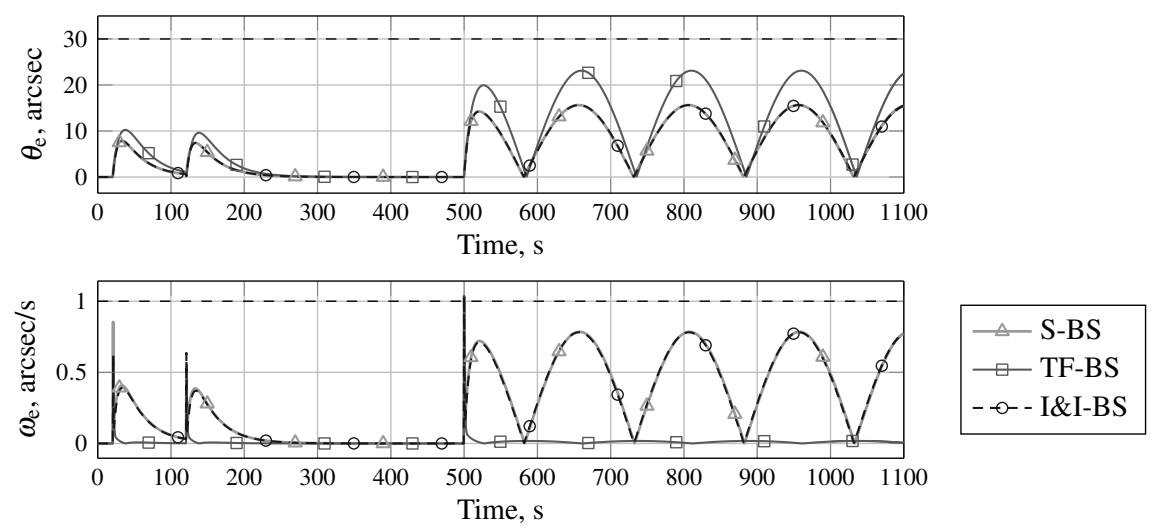

Fig. 2 Pointing and Rate tracking errors in nominal case.

Table 1 Nominal case pointing error RMS.

\begin{tabular}{lccc}
\hline & S-BS & TF-BS & I\&I-BS \\
\hline$\theta_{\mathrm{e}, \mathrm{RMS}}, \operatorname{arcsec}$ & 8.161 & 11.899 & 8.154 \\
\hline
\end{tabular}

Table 2 Nominal case estimation error RMS.

\begin{tabular}{lrr}
\hline & TF-BS & I\&I-BS \\
\hline$\left\|\boldsymbol{\delta}_{\mathbf{m}_{\mathrm{d}}}\right\|_{\mathrm{RMS}}, \mu \mathrm{Nm}$ & 43.203 & 10.137 \\
\hline
\end{tabular}




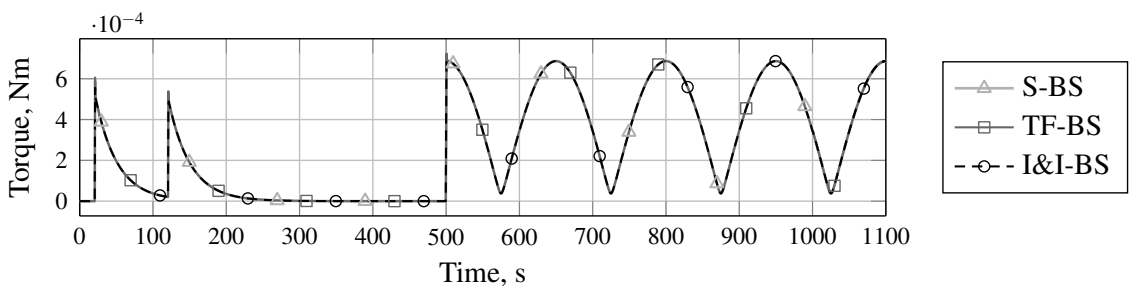

Fig. 3 Actuation torque in nominal case.

Table 3 Total workload in nominal case.

\begin{tabular}{cccc}
\hline & S-BS & TF-BS & I\&I-BS \\
\hline Workload, mJ & 1.5103 & 1.5104 & 1.5103 \\
\hline
\end{tabular}

\section{Uncontrolled Reaction Wheel}

Wheel anomalies are one of the most common GNC-related failures. The Iridium 42 mission, for instance, failed due to a faulty tachometer in one of the wheels, which lead to unknown real actuation [19]. In this test, the effect of an uncontrolled saturated (redundant) reaction wheel is modeled. A $1 \mathrm{~kg}$ wheel is assumed stuck at $4200 \mathrm{rpm}$ producing a strong gyroscopic moment unknown to the control system. Fig. 4 and 5 show that the non-adaptive control cannot cope with such a strong disturbance. The adaptive laws, on the other hand, are quite successful in this case. While the TF-BS controller sees a 54\% pointing degradation (Table 4), the I\&I-BS law is seemingly undisturbed, maintaining virtually the same performance as in the nominal scenario. This is explained by the extremely low disturbance estimation error by this controller (Table 5), which is roughly one order of magnitude better than that of the TF law. The same can be seen in Fig. 6, where the estimation errors of the adaptive laws are plot against the total disturbance (in norm). Note that, in this scenario, the TF-BS controller does not manage to properly track the rate signal, violating both requirement limits. As Fig. 7 shows, the laws' control actions are nearly indistinguishable. The total workload (Table 6), however, tells that the adaptive schemes demand slightly less effort, being the I\&I-BS law the least demanding.

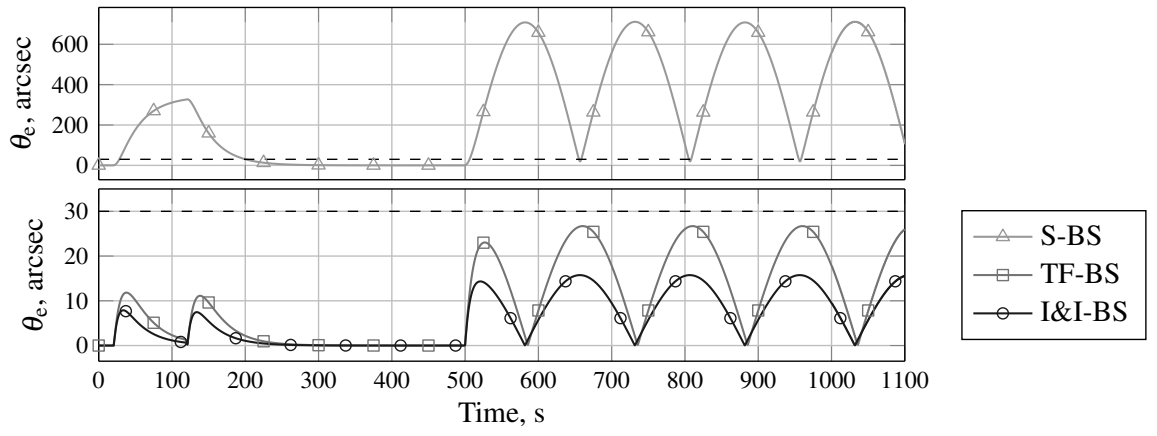

Fig. 4 Pointing error in an uncontrolled reaction-wheel case 


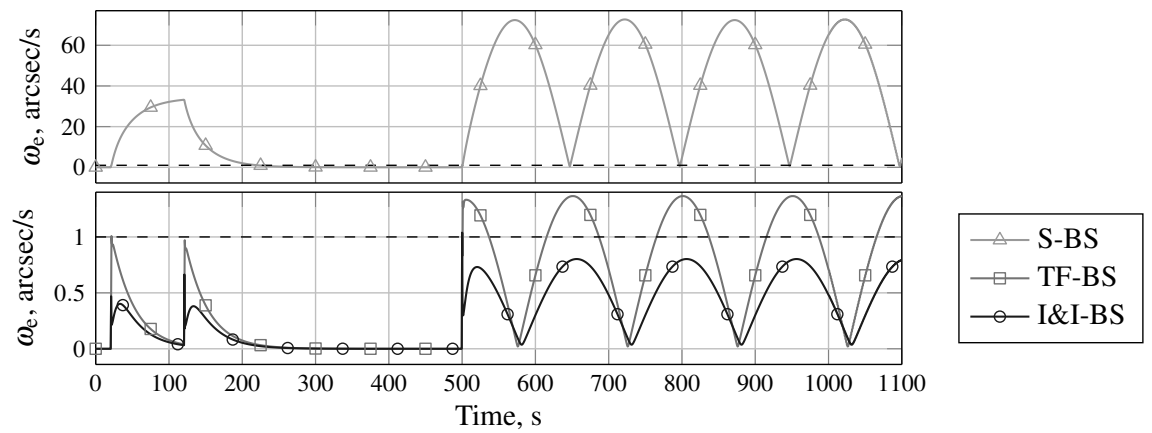

Fig. 5 Rate tracking error in an uncontrolled reaction-wheel case.

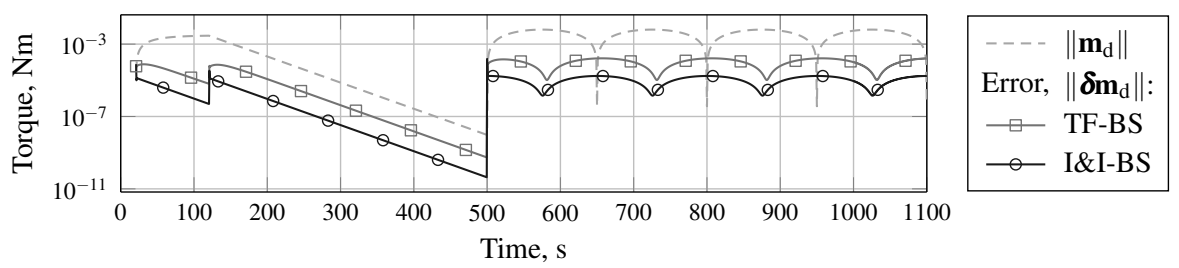

Fig. 6 Total disturbance and estimation error norms in an uncontrolled reaction-wheel case.

Table 4 Pointing error RMS in an uncontrolled reaction-wheel case.

\begin{tabular}{cccc}
\hline$\theta_{\mathrm{e}, \mathrm{RMS}}$ & S-BS & TF-BS & I\&I-BS \\
\hline Value, arcsec & 384.370 & 18.271 & 8.210 \\
Ratio to nominal & 47.099 & 1.536 & 1.007
\end{tabular}

Table 5 Estimation error RMS in the uncontrolled reaction-wheel case.

\begin{tabular}{ccc}
\hline$\left\|\boldsymbol{\delta}_{\mathrm{d}}\right\|_{\mathrm{RMS}}$ & TF-BS & I\&I-BS \\
\hline Value, $\mu \mathrm{Nm}$ & 148.957 & 15.915 \\
Ratio to $\left\|\mathbf{m}_{\mathrm{d}}\right\|_{\mathrm{RMS}}$ & 0.044 & 0.005 \\
\hline
\end{tabular}

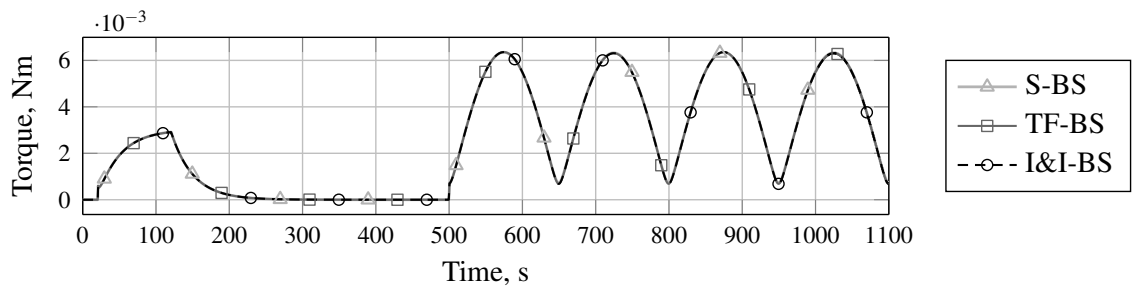

Fig. 7 Actuation torque in an uncontrolled reaction-wheel case.

Table 6 Total workload in an uncontrolled reaction-wheel case.

\begin{tabular}{cccc}
\hline & S-BS & TF-BS & I\&I-BS \\
\hline Workload, mJ & 18.7416 & 18.7251 & 18.7237 \\
\hline
\end{tabular}




\section{Moving Payload}

Many spacecraft instruments/payloads have moving parts. An example is the moving mirror of NASA's Geostationary Imaging Fourier Transform Spectrometer [7].

In the present case, a smaller spectrometer mirror is considered and assumed to be unaccounted for in the control design. The piece, a $0.5 \mathrm{~cm} \times 5 \mathrm{~cm} \times 5 \mathrm{~cm}$ cuboid of $30 \mathrm{~g}$, oscillates about its own center of mass. Its angular orientation is modeled as a sinusoidal function with $15 \mathrm{~s}$ of period and peak-to-peak amplitude of $90 \mathrm{deg}$.

While the required pointing is achieved by the three controllers (Fig. 8), although with obvious oscillations on the part of S-BS and TF-BS, rate tracking proves to be the main difficulty. Only the adaptive schemes comply with the rate requirement. Among these the I\&I is clearly less disturbed, again roughly maintain its nominal performance (Table 7). As Table 8 shows, the estimation deteriorated for both adaptive laws with respect to the previous scenario. As before, the I\&I observer yielded about one order of magnitude lower estimation error than the TF law (Fig. 9). Fig. 10 shows that, as in the two previous cases, control action is very similar among the three controllers. The total workload, displayed on Table 9, reveals that once again the I\&I-BS demanded a notch less control action.
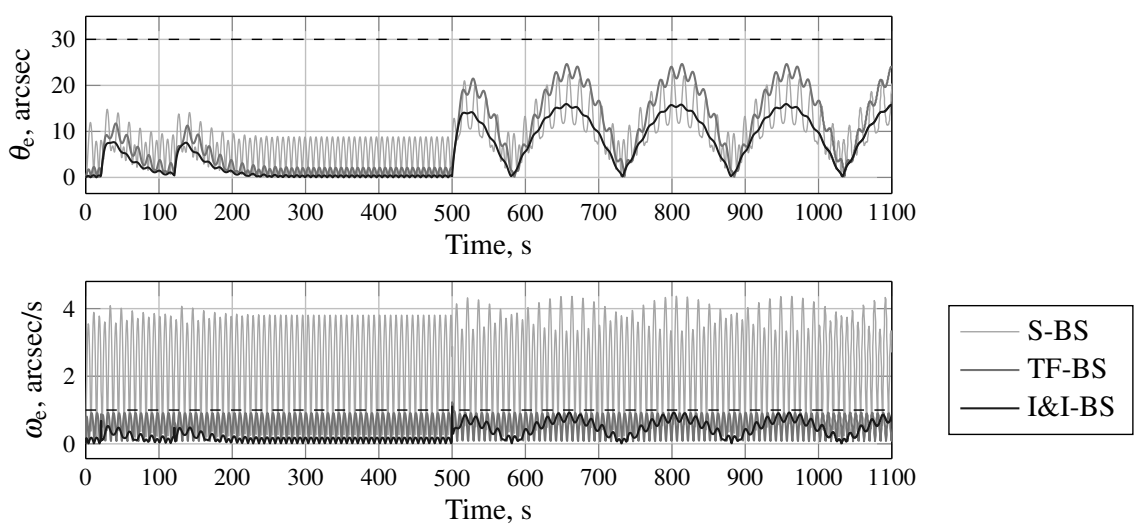

Fig. 8 Pointing and Rate tracking errors in a moving payload case.

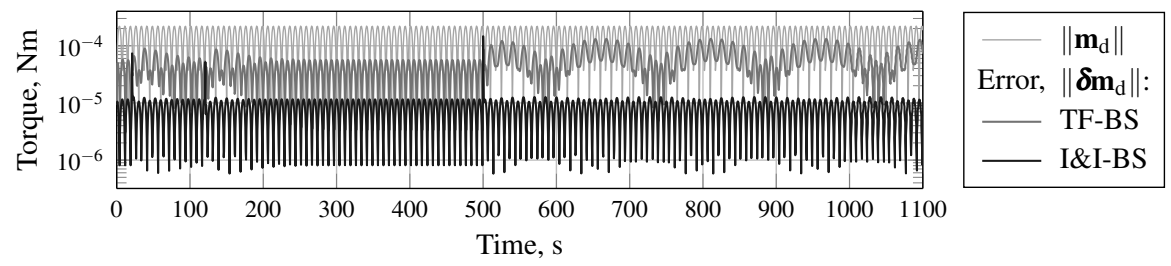

Fig. 9 Total disturbance and estimation error norms in a moving payload case. 
Table 7 Pointing error RMS in a moving payload case.

\begin{tabular}{cccc}
\hline$\theta_{\mathrm{e}, \mathrm{RMS}}$ & $\mathrm{S}-\mathrm{BS}$ & $\mathrm{TF}-\mathrm{BS}$ & $\mathrm{I} \& \mathrm{I}-\mathrm{BS}$ \\
\hline Value, arcsec & 10.280 & 12.210 & 8.161 \\
Ratio to nominal & 1.260 & 1.026 & 1.001 \\
\hline
\end{tabular}

Table 8 Estimation error RMS in a moving payload case.

\begin{tabular}{ccc}
\hline$\left\|\boldsymbol{\delta}_{\mathrm{d}}\right\|_{\mathrm{RMS}}$ & TF-BS & I\&I-BS \\
\hline Value, $\mu \mathrm{Nm}$ & 83.858 & 12.990 \\
Ratio to $\left\|\mathbf{m}_{\mathrm{d}}\right\|_{\text {RMS }}$ & 0.535 & 0.083 \\
\hline
\end{tabular}

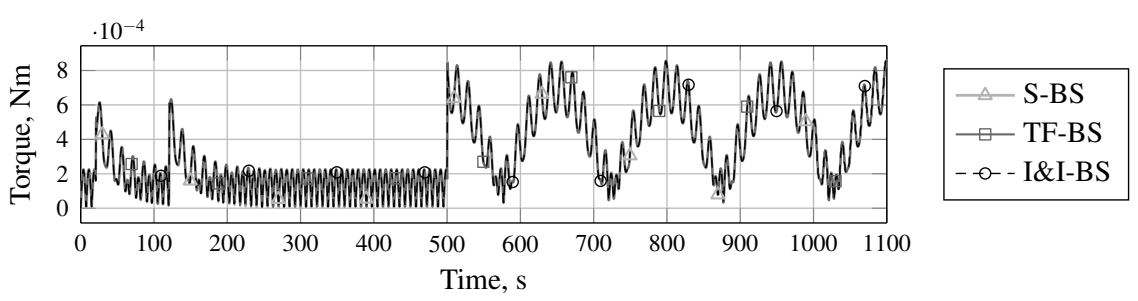

Fig. 10 Actuation torque in a moving payload case.

Table 9 Total workload in a moving payload case.

\begin{tabular}{lccc}
\hline & S-BS & TF-BS & I\&I-BS \\
\hline Workload, mJ & 1.6411 & 1.6394 & 1.6357 \\
\hline
\end{tabular}

\section{Conclusion}

The I\&I adaptive control framework, combined with nonlinear Backstepping, has been used to develop an attitude controller for a micro-satellite. A novel input-tostate stability result for a class of I\&I controlled strict-feedback systems with timevarying uncertainties has been introduced. This allowed a rather general modelling of the rotational dynamics uncertainty. Results for two different severely disturbed cases clearly revealed superior robustness of the I\&I controller in comparison to a baseline standard Backstepping law and to a "traditional" Tuning Functions adaptive scheme. In fact, the I\&I control law managed to virtually keep its nominal performance in all scenarios, having in the disturbance-free case matched the baseline (full-information) law. The tight tracking of the disturbance torque by the I\&I estimator greatly contributed to this result. Although the differences in control torque were marginal in all cases, the proposed I\&I law demanded the least control effort.

\section{References}

[1] A. Astolfi and R. Ortega. "Immersion and Invariance: A New Tool for Stabilization and Adaptive Control of Nonlinear Systems". In: IFAC Symposium on Nonlinear Control System Design. 2001.

[2] A. N. Atassi and H. K. Khalil. "A Separation Principle for the Stabilizationof a Class of Nonlinear Systems". In: IEEE Transactions on Automatic Control 44.9 (1999), pp. 16721687. 
[3] J. A. Farrell, M. Polycarpou, and M. Sharma. "Adaptive Backstepping with Magnitude, Rate, and Bandwidth Constraints: Aircraft Longitude Control". In: Proceedings of the Annual American Control Conference. 2003.

[4] J. A. Farrell et al. "Command Filtered Backstepping". In: IEEE Transactions on Automatic Control 54 (2009), pp. 1391-1395.

[5] E. Gill et al. "Atmospheric Aerosol Characterization with the Dutch-Chinese FAST Formation Flying Mission”. In: Acta Astronautica 66 (2010), pp. 1044-1051.

[6] J. Guo et al. "Status of the FAST Mission: Micro-Satellite Formation Flying for Technology, Science and Education". In: 60th International Astronautical Congress. 2009.

[7] J Juang. Instrument Attitude Precision Control. Tech. rep. Langley Research Center, Virginia: NASA, 2004.

[8] J. L. Junkins and P. Singla. "How Nonlinear Is It? A Tutorial on Nonlinearity of Orbit and Attitude Dynamics". In: Journal of Astronautical Sciences 52 (2004), pp. 7-60.

[9] I. Kanellakopoulos, P. Kokotović, and A. S. Morse. "Systematic Design of Adaptive Controllers for Feedback Linearizable Systems". In: IEEE Transactions on Automatic Control 36.11 (1991), pp. 1241-1253.

[10] D. Karagiannis and A. Astolfi. "Nonlinear adaptive control of systems in feedback form: An alternative to adaptive Backstepping”. In: Systems \& Control Letters 57 (2008), pp. 733-739.

[11] D. Karagiannis and A. Astolfi. "Nonlinear Observer Design Using Invariant Manifolds and Applications". In: 44th IEEE Conference on Decision and Control. 2005.

[12] D. Karagiannis, D. Carnevale, and A. Astolfi. "Invariant Manifold Based Reduced-Order Observer Design for Nonlinear Systems". In: IEEE Transactions on Automatic Control 53.11 (2008), pp. 2602-2614.

[13] H. K. Khalil. Nonlinear Systems. Third. Prentice Hall, 2002.

[14] P. Kokotović and M. Arcak. "Constructive Nonlinear Control: A Historical Perspective". In: Autimatica 37 (2001), pp. 637-662.

[15] M. Krstić, I. Kanellakopoulos, and P. V. Kokotović. Nonlinear and Adaptive Control Design. Wiley-Interscience, 1995.

[16] C. Li and G. Ma. "Adaptive Backstepping Control for Attitude Tracking of a Spacecraft". In: IEEE International Symposium on Industrial Electronics. 2007.

[17] D. Maessen et al. "Mission Design of the Dutch-Chinese FAST Micro-Satellite Mission". In: 7th IAA Symposium on Small Satellites for Earth Observation. 2009.

[18] J. Pomet and L. Praly. "Adaptive Nonlinear Regulation: Estimation from the Lyapunov Equation”. In: IEEE Transactions on Automatic Control 37 (1992), pp. 729-740.

[19] B. Robertson and E. Stoneking. "Satellite GN\&C Anomaly Trends". In: 26th Annual AAS Guidance and Control Conference. 2003.

[20] H. Schaub and J. L. Junkins. "Stereographic Orientation Parameters for Attitude Dynamics: A Generalization of the Rodrigues Parameters". In: Journal of the Astronautical Sciences 44 (1996), pp. 1-19.

[21] M. D. Shuster. “A Survey of Attitude Representations”. In: The Journal of Astronautical Sciences 41.4 (1993), pp. 439-517.

[22] L. Sonneveldt et al. "Immersion and Invariance Based Nonlinear Adaptive Flight Control". In: AIAA Guidance, Navigation and Control Conference and Exhibit. 2010.

[23] G. F. Trigo. "Robust and Adaptive Nonlinear Attitude Control of a Spacecraft: A Comparison of Backstepping-based Designs". MA thesis. Delft University of Technology, 2011.

[24] P. Tsiotras. "New Control Laws for the Attitude Stabilization of Rigid Bodies". In: IFAC Symposium on Automatic Control in Aerospace. 1994.

[25] E.R. van Oort et al. "Full Envelope Modular Adaptive Control of a Fighter Aircraft using Orthogonal Least-Squares". In: Journal of Guidance, Navigation and Dynamics 33.5 (2010), pp. 1461-1472.

[26] B. Wie. Space Vehicle Dynamics and Control. Ed. by J. A. Schetz. Second. AIAA, 2008.

[27] W. E. Wiesel. Spaceflight Dynamics. Second. Irwin/McGraw-Hill, 1997.

[28] P. P. Yip and J. K. Hedrick. "Adaptive Dynamic Surface Control: A Simplified Algorithm for Adaptive Backstepping Control of Nonlinear Systems". In: International Journal of Control 71 (1998), pp. 959-979. 